J. Clin. Chem. Clin. Biochem.

Vol. 15, 1977, pp. 583-585

\title{
Vergleichende Harnstoffbestimmung mit dem Merckognost Teststäbchen und einer photometrischen Methode
}

\author{
Von $A$. Scholer, A. Pianezzi, D. Vonderschmitt \\ Klinisch-chemisches Laboratorium, Zentrallabor des Kantonsspitals, Basel, Schweiz, und \\ W. Götz
}

Wissenschaftliche Dokumentation, E. Merck, Darmstadt

(Eingegangen am 10. September 1976/23. Juni 1977)

Zusammenfassung: Genauigkeit und Praktikabilität des Teststäbchens Merckognost-Harnstoff wurden an Plasma, Hämolysat, Kapillar- und Venenblut überprüft. Als Referenzmethode diente das Diacetylmonoxim-Verfahren am AutoAnalyzer II. Ein möglicher Einfluß unterschiedlicher Hämatokritwerte auf die mit Vollblut erhaltenen Ergebnisse wurde in die Untersuchungen einbezogen.

Die vergleichenden Untersuchungen ergaben eine ausgezeichnete Ubereinstimmung der mit beiden Verfahren erhaltenen Werte. Der Einfluß unterschiedlicher Hämatokritwerte auf die mit dem Teststäbchen in Vollblut erzielten Ergebnisse erwies sich als vernachlässigbar. Die Brauchbarkeit des Teststäbchen-Systems sowohl für die Routine als auch für die Notfalldiagnostik wird bestätigt.

\section{Determination of urea with the Merckognost test strips, and a comparison with a photometric method}

Summary: The precision and practicability of the Merckognost-Urea Test Strips was tested in plasma, haemolysate, capillary and venous blood. The diacetylmonoxim method using an AutoAnalyzer II was taken as the reference method. The possibility of varying haematocrit values influencing the whole blood findings was taken into consideration in the investigations.

The comparative investigation showed that the values obtained by the two different methods correspond very well. The influence of varying haematocrit values on the results obtained with the test strips in whole blood proved negligible. Confirmation is provided of the usefulness of the test strip system both in routine procedures and also in emergency diagnoses.

\section{Einführung}

Da die Harnstoffbestimmung in der Notfalldiagnostik wichtig ist und häufig ein Test im Vollblut nützlich wäre, prüften wir einen Teststreifen zur quantitativen Messung vọn Harnstoff in Plasma, Vollblut und Hämolysat und verglichen die Ergebnisse mit der Diacetylmonoxim-Methode auf dem Auto-Analyzer II (1). Die Härnstoff fmessung auf dem Teststreifen beruht auf der Ureasemethode.

\section{Methodik}

\section{Material}

Für die Untersuchung von Plasma verwendeten wir Proben aus dem bei den täglichen Routịneanalysen anfallenden Material. Bei der vergleichenden. Prüfung von Plasma- und Kapillarblut- proben stammte das Plasma ebenfalls aus der täglichen Routine. Den entsprechenden Patien ten entnahmen wir am gleichen Tag aus der Fingerbeere Kapillarblut.

Die Entnahme von Venenblut für die Analysen im Vollblut erfolgte mit dem Vacutainer, der als Gerinnungshemmer Lithiumheparinat enthält. Für die vergleichenden Unter suchungen in Vollblut, Hämolysat und Plasma wurden Plasma und Vollblut im Verhältnis 2:1 mit physiologischer $\mathrm{NaCl}$-Lösung verdünnt. Zur Herstellung von Hämolysat wưrde das Blut im Verhältnis 2:1 mit destilliertem Wasser verdünnt und die Probe zentrifugiert.

\section{Reagenzien und Geräte}

Die Merckognost-Harnstoff Teststäbchen, Merck Art.-Nr. 11001 , tragen eine Reaktionszone und eine davon getrennte Indikatorzone. Die Reaktionszone enthält Urease, die Indikatorzone eine Säure und einen pH-Indikator. Der in der Analysenprobe enthaltene Harnstoff wird in der Reaktionszone spezifisch in Kohlendioxid und Ammoniak gespalten. Das Ammoniak diffundiert zur Indikatorzone, wo es einen Farbumschlag von Gelb nach 
Blau bewirkt. Die Länge der entstehenden Blauzone ist von der Harnstoffkonzentration, der Temperatur und der Diffusionszeit abhängig. Auswertetabellen für die verschiedenen möglichen Modifikationen der Bestimmung (unterschiedliche Ablesezeit, unterschiedliches Probenmaterial) sind in der Packung enthalten. Es kamen Stäbchen aus verschiedenen Chargen zum Einsatz.

Die Hämatokritwerte wurden mit dem Coulter Counter S ermittelt.

Durchführung der Untersuchungen

Die Harnstoffbestimmungen erfolgten unter Routinebedingungen. Die Merckognost-Harnstoff Teststäbchen wurden entsprechend der Gebrauchsanweisung der Herstellerfirma angewandt. Die Raumtemperatur lag zwischen 22 und $25^{\circ} \mathrm{C}$, so daß eine Korrektur der Ergebnisse mit Temperaturfaktoren (s. dazu unter "Diskussion") nicht notwendig war.

Soweit nicht anders angegeben, betrug die Reaktionszeit $30 \mathrm{~min}$. Die Ablesung der Ergebnisse nahmen Personen vor, die nicht besonders fuir diese Aufgabe geschult waren (Auswertung siehe Vorschrift des Herstellers).

Die vorgenommenen Vergleiche können Tabelle 1 entnommen werden.

Zur Prüfung der Richtigkeit des Teststäbchens MerckognostHarnstoff in der Notfalldiagnostik untersuchten wir 33 Vollblutproben nach $10 \mathrm{~min}$. Die Kontrolle erfolgte durch Bestimmung des Harnstoffwertes in der gleichen Blutprobe wieder mit dem Diacetylmonoxim-Verfahren.

Um einen möglichen Einfluß unterschiedlicher Hämatokritwerte festzustellen, wurden im Verlauf von etwa 3 Monaten an insgesamt 145 Blutproben die Harnstoff werte mit MerckognostTeststäbchen in Plasma und Vollblut und mit dem Diacetylmonoxim-Verfahren im Plasma gemessen und von jeder Blutprobe der Hämatokritwert bestimmt.

Die statistische Auswertung der Resultate erfolgte durch Berechnung der Regressionsgeraden, des Korrelations- und des Variationskoeffizienten (2)

\section{Ergebnisse}

Tabelle 1 faßt die Ergebnisse zusammen und bestätigt die gute Übereinstimmung.

Die Korrelation der mit beiden Methoden an einer Serie von 119 Plasmen erhaltenen Ergebnisse zeigt Abbil- dung 1, die Korrelation der Ergebnisse von 99 Kapillarblutproben, die mit Teststreifen untersucht wurden, und den in den Plasmen derselben Patienten mit der Diacetylmonoximmethode am AutoAnalyzer II erhaltenen ist in Abbildung 2 dargestellt.

Die Werte der Vergleiche lagen für alle Serien im Bereich von 0 bis $30 \mathrm{mmol} / \mathrm{l}$ (pathologisch über $6,66 \mathrm{mmol} / \mathrm{l}$, siehe auch Abb. 1 und 2).

Beim Vergleich der mit den Merckognost-Harnstoff Teststäbchen in der Notfalldiagnostik nach 10 min erhaltenen Werte mit den entsprechenden mit der Diacetylmonoxim-Methode erhaltenen Werte war $r=0,890$. Vergleicht man diese Korrelation mit den oben angegebenen,

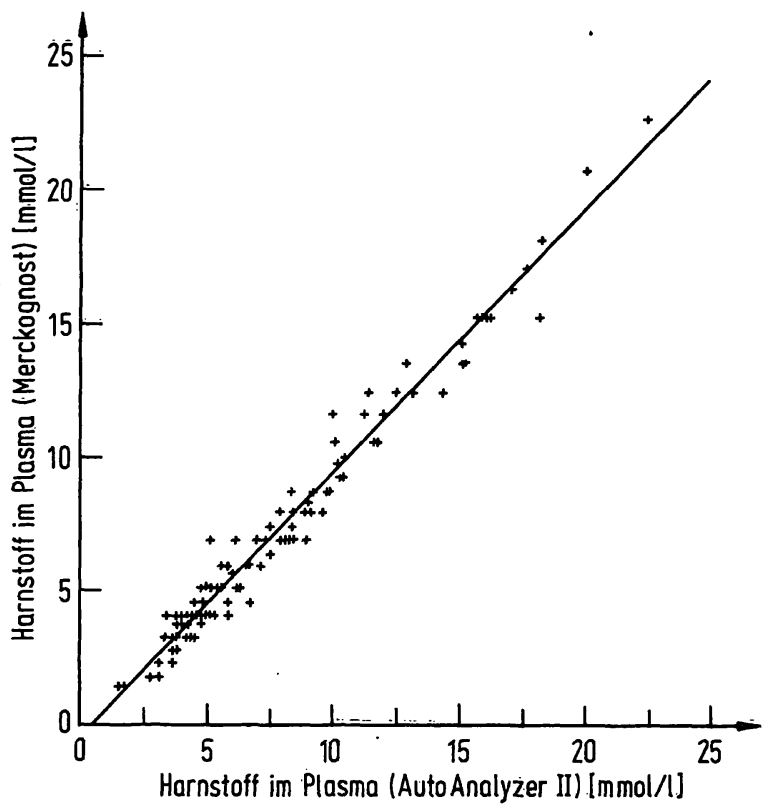

Abb. 1. Auswertung der bei der vergleichenden Untersuchung von 119 Plasmaproben mit Merckognost-Harnstoff und der Diacetylmonoxim-Methode erhaltenen Resultate. $\mathrm{N}=119 ; \overline{\mathrm{x}}=7,80 \overline{\mathrm{y}}=7,23 ; \mathrm{y}=-0,382+0,976 \mathrm{x}$

Tab. 1. Korrelation, Regression und Variationskoeffizienten der beiden Methoden (TS = Teststreifen, AA II = DiacetylmonoximMethode am AutoAnalyzer II) bei Verwendung verschiedener P̈robenmaterialien.

\begin{tabular}{|c|c|c|c|c|}
\hline Material & Anzahl & $\begin{array}{l}\text { Korrelation } \\
\mathbf{r}\end{array}$ & $\begin{array}{l}\text { Regression } \\
y\end{array}$ & $\begin{array}{l}\text { VK } \\
{[\%]}\end{array}$ \\
\hline $\begin{array}{l}\text { Plasma (TS)/Plasma (AA II)* } \\
\text { Plasma (TS)/Plasma (AA II) } \\
\text { Plasma (TS)/Plasma (AA II) }\end{array}$ & $\begin{array}{r}119 \\
99 \\
42\end{array}$ & $\begin{array}{l}0,985 \\
0,980 \\
0,984\end{array}$ & $\begin{array}{r}-0,382+0,976 x \\
0,057+0,968 x \\
0,313+0,972 x\end{array}$ & $\begin{array}{r}9,3 \\
18,3 \\
20,8\end{array}$ \\
\hline $\begin{array}{l}\text { Kapillarblut (TS)/Plasma (AA II) } \\
\text { Vollblut (TS)/Plasma (AA II) } \\
\text { Kapillarblut (TS)/Plasma (AA II)** }\end{array}$ & $\begin{array}{l}38 \\
42 \\
99\end{array}$ & $\begin{array}{l}0,990 \\
0,980 \\
0,989\end{array}$ & $\begin{array}{l}0,266+0,989 x \\
0,387+0,958 x \\
0,071+0,975 x\end{array}$ & $\begin{array}{r}8,4 \\
23,8 \\
13,6\end{array}$ \\
\hline $\begin{array}{l}\text { Plasma (TS)/Kapillarblut (TS) } \\
\text { Plasma (TS)/Vollblut (TS) } \\
\text { Plasma (TS)/Kapillarblut (TS) }\end{array}$ & $\begin{array}{r}35 \\
59 \\
100\end{array}$ & $\begin{array}{l}0,979 \\
0,980 \\
0,984\end{array}$ & $\begin{array}{l}0,446+1,029 x \\
0,161+0,973 x \\
0,171+0,971 x\end{array}$ & $\begin{array}{l}11,6 \\
19,9 \\
16,3\end{array}$ \\
\hline Vollblut (TS)/Vollblut (AA II) & 59 & 0,989 & $0,132+1,007 x$ & $15 ; 6$ \\
\hline $\begin{array}{l}\text { Hämolysat (TS)/Plasma (TS) } \\
\text { Hämolysat (TS)/Vollblut (TS) } \\
\text { Hämolysat (TS)/Hämolysat (AA II) }\end{array}$ & $\begin{array}{l}59 \\
59 \\
59\end{array}$ & $\begin{array}{l}0,979 \\
0,995 \\
0,993\end{array}$ & $\begin{array}{l}0,267+0,256 x \\
0,123+0,980 x \\
0,092+0,955 \times\end{array}$ & $\begin{array}{r}20,2 \\
9,3 \\
11,1\end{array}$ \\
\hline
\end{tabular}

* siehe auch Abbildung 1

** siehe auch Abbildung 2 


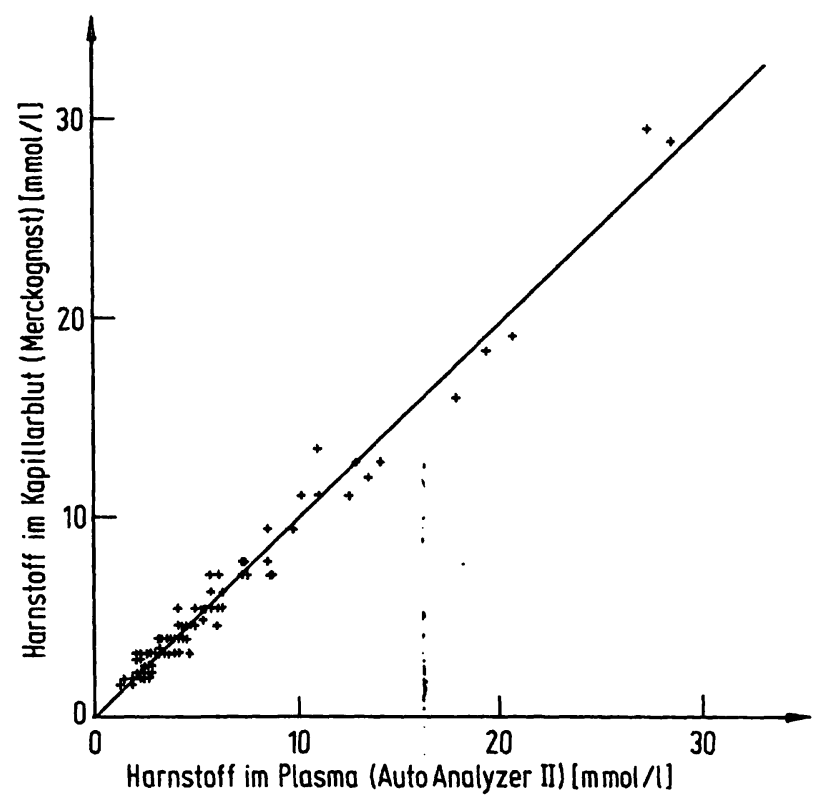

Abb. 2. Auswertung der an 99 Proben mit der DiacetylmonoximMethode im Plasma und mit Merckognost-Harnstoff im Kapillarblut erhaltenen Werte.

$N=99 ; \bar{x}=5,37, \bar{y}=5,31 ; y=0,071+0,975 x$

bei der vom Hersteller empfohlenen Reaktionszeit von 30 min erhaltenen Resultaten, so ergibt sich, daß die Präzision der Ergebnisse mit der Länge der Reaktionszeit zunimmt. Für den Notfall, in dem es in erster Linie darauf ankommt, schnell festzustellen, ob überhaupt eine Azotämie vorliegt, reicht die mit den MerckognostHarnstoff Teststäbchen nach 10 min erhaltene Präzision jedoch völlig aus. Die gemessenen Konzentrationen lagen zwischen etwa 1,7 und 61,6 mmol Harnstoff/1.

Die Prüfung eines möglichen Einflusses unterschiedlicher Hämatokritwerte auf die mit den Merckognöst-Harnstoff Teststäbchen in Vollblut erzielten Ergebnisse ergab, daß hohe Hämatokritwerte geringere Harnstoffwerte vortäuschen. Umgekehrt werden bei niederen Hämatokritwerten zu hohe Resultate für den Harnstoff

\section{Literatur}

1. Technicon Bulletin 01/1970.

2. Richterich, R. (1971), Klinische Chemie - Theorie und Praxis. 3. Aufl., S. 44-49, Verlag S. Karger, Basel-München-Paris-London-New York-Sydney. erhalten. Die hierdurch bedingte Verfälschung der Ergebnisse ist jedoch für die Praxis unbedeutend, da die Abweichungen viel geringer sind, als die allgemeine Streuung der Methode.

\section{Diskussion}

Die in der Literatur (3) angegebene gute Úbereinstimmung zwischen den Resultaten, die mit den Merckognost-Harnstoff Teststäbchen und denen, die mit der Diacetylmonoxim-Methode erhalten werden, konnte durch die sehr guten Korrelationen bei den beschriebenen Versuchsanordnungen bestätigt werden. Das Verfahren mit Merckognost-Harnstoff ist für die Klinik vor allem für die Abteilungen von großem Nutzen, bei denen es schwierig ist, von den Patienten Venenblut zu erhalten, z. B. bei Kindern und bei der Uberwachung von Patienten mit einer Azotämie. Sowohl für die Klinik als auch für die Praxis bringt die Anwendung des Teststäbchens in der Notfalldiagnostik, z. B. bei der differentialdiagnostischen Abklärung eines Komas unklarer Genese, eine erhebliche Erleichterung. Den größten Nutzen dürfte von diesen Teststäbchen der Arzt in der Praxis haben, der damit ohne großen Laboraufwand schnell den Wert für einen wichtigen Parameter zur Beurteilung der Nierenfunktion seiner Patienten erhalten kann.

Für den Einsatz der Merckognost-Harnstoff Teststäbchen in der Routine ist neben der Anwendbarkeit in unterschiedlichem Probenmaterial (Kapillar- und Venenblut, Plasma, Serum und Hämolysat) von Vorteil, daß durch Anwendung von Temperaturkorrekturfaktoren die Reaktion in einem Bereich von $18-28^{\circ} \mathrm{C}$ Umgebungstemperatur durchgeführt werden kann. Es wird allerdings empfohlen, vor der Übernahme des Tests in die Routine einige Erfahrungen mit dem System zu sammeln. Die genaue Einhaltung der Vorschrift ist Voraussetzung für gute Ergebnisse. Eine eventuell auftretende Hämolyse stört nicht. Der Einfluß unterschiedlicher Hämatokritwerte kann vernachlässigt werden.
3. Dunsbach, F. \& Seiffert, U. B. (1974), Med. Labor. 27, 263-265.
A. Scholer dipl. chem. Klin.-Chem. Laboratorium Kantonsspital Basel CH-4031 Basel 
